\title{
Prediction of Damping Property and Design for Optimization of Magnetic Rubber Damper with Constraining Layer (MRDC)
}

\author{
Masanori HANSAKA \\ Senior Researcher, Vibration-Isolation Materials Group \\ Materials Technology Division
}

\begin{abstract}
The magnetic rubber damper with a constraining layer (MRDC), which we developed, is easy to install on steel vibrating objects and has proven to be an excellent vibration damper. The damping property of an MRDC is substantially attributable to the frictional loss between its magnetic rubber layer and a steel object. The authors devised a theory to predict its damping properties by taking friction into consideration, the theory itself being based on an analysis of the flexural vibration of a beam with MRDC. The accuracy of the proposed theory has been verified by comparing analytical and experimental results, and a design to optimize MRDCs has been determined from those obtained from calculation and measurement.
\end{abstract}

Keywords: magnetic, vibration damper, butyl rubber, flexural vibration, loss factor

\section{Introduction}

Train speeds have been increasing in recent years, and this is just one of the reasons why the development of effective methods to control vibration and noise is becoming increasingly more in demand on railways. The installation of vibration dampers is one of the most effective methods for controlling noise and vibration, whereas conventional dampers have one major drawback in that they are very labor intensive when being installed. This is because they are fitted to vibrating objects with an adhesive, which requires a number of process stages, for example, preparatory treatment that includes cleaning, blasting and coating of base layers with primer. To solve this problem, we have developed an innovative type of vibration damper, a magnetic rubber damper with a constraining layer (MRDC) ${ }^{1)}$. This consists of a magnetic rubber layer, made of butyl rubber mixed with ferrite powder and then magnetized, and a steel constraining layer. Because it is magnetized, MRDC is easy to install on vibrating steel objects such as railway steel bridges.

It is a characteristic of MRDC that the magnetic rubber layer slides on the vibrating base layer, generating frictional loss between them. In order to devise a theory to predict its damping properties, a basic one-dimensional analysis of the flexural vibration of a beam with MRDC is implemented by taking into consideration a sliding displacement and a frictional loss between the magnetic rubber and base layers. Moreover, the accuracy of the theory thus devised has been verified by comparing it with experimental data obtained by a resonance method. A design for optimization of MRDC has been also determined, based on results obtained from calculation and measurement.

\section{Flexural vibration analysis ${ }^{2)} 3$ )}

\subsection{Derivation of vibration equations}

This paper presents an outline of the analysis used to predict the damping properties of MRDC, more details of which are described in references 2) and 3).

Hamilton's principle expanded into the non-preservative system given by the following equation should be applied as the kinetic energy principle of MRDC, because MRDC has frictional loss between the magnetic rubber and base layers.

$$
\int_{t_{0}}^{t_{1}}\left(\delta T-\delta P+\sum_{r=1}^{n} Q_{r} \delta q_{r}\right) d t=0
$$

where, $T$ is the kinetic energy; $P$ is the strain energy of three layers, those are the base, magnetic rubber and constraining layers; $Q_{r}$ is the generalized force that works in the non-preservative system; $q r$ is the displacement in the generalized coordinate system; and $t_{0}$ and $t_{1}$ are the time factors when motion is presumed in this system. The subscript $r$ shows the number of points where the generalized force is working. Equation (1) is the original principle used to derive vibration equations.

First, the strain energy $P$ of the three layers is thought as explained below. The beam covered with MRDC is assumed to deform as shown in Fig. 1. $\varepsilon_{i}(x, \zeta, t)(i=1,3)$, that is the strain of the base layer or the constraining layer at a point where the location value along the $x$-axis is $x$ and the distance from the center plane along the $z$-axis is $\zeta$, is given by the following equation, when the displacement of the base layer on the center plane in the $x$-direction is defined as $u_{1}(x, t)$, that of the constraining layer as $u_{3}(x, t)$, and the displacement in the $z$-direction as $w(x, t)$ :

$$
\varepsilon_{i}(x, \zeta, t)=\frac{\partial u_{i}(x, t)}{\partial x}-\zeta \frac{\partial^{2} w(x, t)}{\partial x^{2}}
$$

The shear strain of the magnetic rubber layer $\gamma(x, t)$ is given by the following equation, taking its deformation shown in Fig. 1 into consideration.

$$
\gamma(x, t)=s \gamma^{a}(x, t)
$$
where $\gamma^{a}(x, t)$ is the shear strain of the rubber layer when it is bonded to the base layer. Now, $\gamma$ and $\gamma^{a}$ are considered to be independent of $\zeta$. In addition, s or the ratio of $\gamma$ to $\gamma^{a}$ is considered to be independent of time 


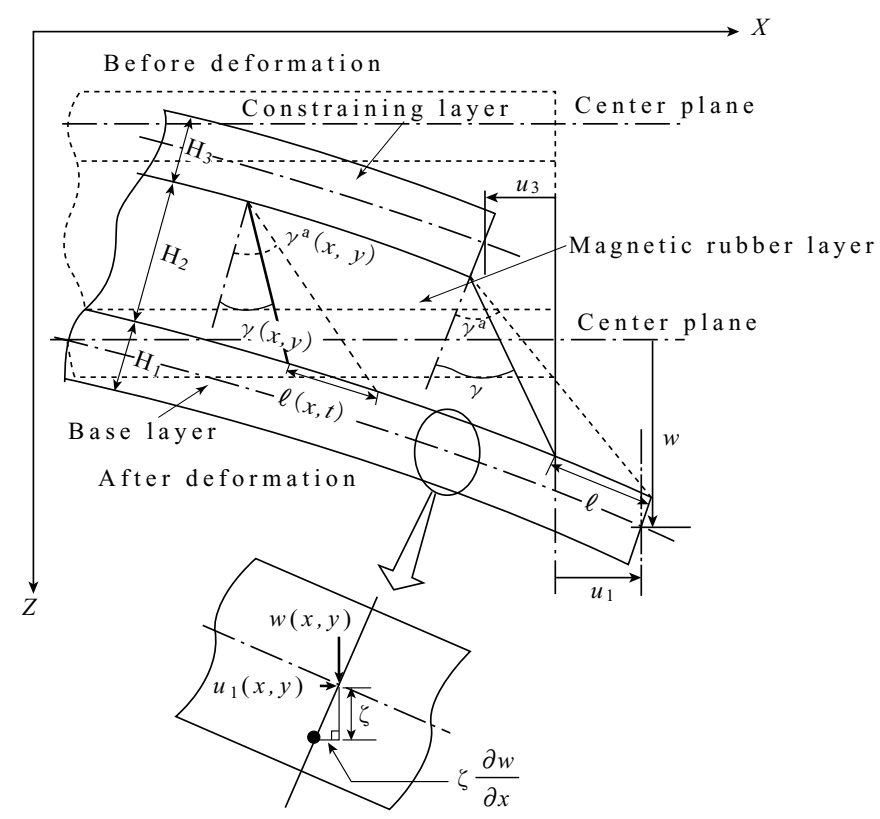

Fig. 1 Deformation of a beam with MRDC

and location since the magnetic attractive force is near constant all over the magnetic rubber layer because the density of mixed ferrite powder is almost uniform and varies little with time.

$\gamma^{a}(x, t)$ is given by the following equation when the geometric relationship shown in Fig. 1 is referred to:

$$
\gamma^{a}(x, t)=\frac{1}{H_{2}}\left[u_{1}(x, t)-u_{3}(x, t)+h \frac{\partial w(x, t)}{\partial x}\right]
$$

where $h=H_{2}+\left(H_{1}+H_{3}\right) / 2$, and $H_{1}, H_{2}, H_{3}$ are the thicknesses of base, magnetic rubber and constraining layers, respectively.

By applying equations (2) to (4), the strain energy $P$ of the three layers can be given by the following equation:

$$
P=P_{1}+P_{2}+P_{3}
$$

where $P_{i}(i=1$ to 3$)$ are the strain energy of the base, magnetic rubber and constraining layers, respectively, given by the following equations:

$$
\begin{aligned}
& P_{1}=\frac{1}{2} E_{1} \int_{0}^{L} \int_{-H_{1} / 2}^{H_{1} / 2} \varepsilon_{1}^{2} d \zeta d x=\frac{1}{2} \int_{0}^{L}\left[K_{1}\left(\frac{\partial u_{1}}{\partial x}\right)^{2}+D_{1}\left(\frac{\partial^{2} w}{\partial x^{2}}\right)^{2}\right] d x \\
& P_{2}=\frac{1}{2} G_{2} \int_{0}^{L} \int_{-H_{2} / 2}^{H_{2} / 2} \gamma^{2} d \zeta d x=\frac{G_{2} s^{2}}{2 H_{2}} \int_{0}^{L}\left(u_{1}-u_{3}+h \frac{\partial w}{\partial x}\right)^{2} d x \\
& P_{3}=\frac{1}{2} E_{3} \int_{0}^{L} \int_{-H_{3} / 2}^{H_{3} / 2} \varepsilon_{3}^{2} d \zeta d x=\frac{1}{2} \int_{0}^{L}\left[K_{3}\left(\frac{\partial u_{3}}{\partial x}\right)^{2}+D_{3}\left(\frac{\partial^{2} w}{\partial x^{2}}\right)^{2}\right] d x
\end{aligned}
$$

where $D_{1}$ and $D_{3}$ are the flexural moduli of the base or constraining layers; $D_{1}=E_{1} H_{1}{ }^{3 / 12}$ and $D_{3}=E_{3} H_{3}{ }^{3} / 12$, in which $E_{1}$ and $E_{3}$ are the Young's modulus of the base or constraining layers; $G_{2}$ is the shear modulus of the magnetic rubber layer; $K_{1}$ and $K_{3}$ are the elongation moduli $\left(K_{1}=E_{1} H_{1}, K_{3}=E_{3} H_{3}\right)$; L is the length of the base layer and is also assumed to be the same with that of constraining layer.

Subsequently, the third term in equation (1) is examined. The generalized force $Q_{r}$ is given as a frictional resistance generated on $d x$, or an infinite distance, and $q_{r}$, or the placement in the generalized coordinate system, is given as the sliding friction $\ell(x, t)$, since it is thought that the frictional loss is given by the product [frictional resistance] $\times$ [sliding displacement]. $\quad \ell(x, t)$ is given by the following equation when the geometric relationship shown in Fig. 1 is referred to:

$$
\ell(x, t)=H_{2}\left(\gamma^{a}-\gamma\right)=(1-s)\left(u_{1}-u_{3}+h \frac{\partial w}{\partial x}\right)
$$

The third term given by a sum of discrete values is converged into an integral calculation shown in the following equation, when $d x$ is divided into infinitely small increments:

$\lim _{n \rightarrow \infty} \sum_{r=1}^{n} Q_{r} \delta q_{r}=-\int_{0}^{L} \operatorname{sgn}\left(\frac{\partial \gamma}{\partial t}\right) \mu F_{M} \times(1-s) \delta\left(u_{1}-u_{3}+h \frac{\partial w}{\partial x}\right) d x$

where $\mu$ is the frictional coefficient between the magnetic rubber and base layers; $F_{M}$ is the magnetic attractive force per unit area; and $\operatorname{sgn}()$ is a function which returns the same sign as that of the input value.

Kinetic energy is given by the following equation:

$$
T=\frac{1}{2} \rho H \int_{0}^{L}\left(\frac{\partial w}{\partial t}\right)^{2} d x
$$

where $\rho$ is the average of the density of the three layers and $H=H_{1}+H_{2}+H_{3}$.

The following vibration equations have been derived after some manipulation by substituting equations (5), (8) and (9) for equation (1):

$$
\begin{aligned}
& D \frac{\partial^{4} w}{\partial x^{4}}-\frac{G_{2} h^{2}}{H_{2}}\left(\frac{\partial u_{1}}{\partial x}-\frac{\partial u_{3}}{\partial x}+h \frac{\partial^{2} w}{\partial x^{2}}\right)+\rho H \frac{\partial^{2} w}{\partial t^{2}}=0 \\
& K_{1} \frac{\partial^{2} u_{1}}{\partial x^{2}}-\frac{G_{2} s^{2}}{H_{2}}\left(u_{1}-u_{3}+h \frac{\partial w}{\partial x}\right)-\operatorname{sgn}\left(\frac{\partial \gamma}{\partial t}\right) f=0 \\
& K_{3} \frac{\partial^{2} u_{3}}{\partial x^{2}}+\frac{G_{2} s^{2}}{H_{2}}\left(u_{1}-u_{3}+h \frac{\partial w}{\partial x}\right)+\operatorname{sgn}\left(\frac{\partial \gamma}{\partial t}\right) f=0
\end{aligned}
$$

where $D=D_{1}+D_{3}$ and $f=\mu F_{M}(1-\mathrm{s})$.

In these equations, $K_{1}, K_{3}$ and $G_{2}$ are regarded as real values in consideration of the fact that the inner losses of three layers are considered to be small. In addition, $\mathrm{s}$ the ratio of $\gamma$ to $\gamma^{a}$, dynamic frictional coefficient $\mu$ and magnetic attractive force FM are measured by the methods described in reference 4 ).

\subsection{Solution of the vibration equations}

\subsubsection{Substituting frictional resistance by viscous resis-} tance

The derived equations (10a) to (10c) are difficult to solve because the terms of frictional resistance involved are nonlinear. We therefore tried to solve these equations by substituting frictional resistance by linear viscous resistance, the energy of which is the same as the frictional loss. This is shown in the following equation, when the equivalent viscous coefficient per unit area is denoted as $C_{e q}$, and viscous resistance is considered to be proportional to the first-order differential coefficient of $\ell(x, t)$ with respect to $t$ :

$$
\operatorname{sgn}\left(\frac{\partial \gamma}{\partial t}\right) \mu F_{M}=C_{e q}(1-s) \frac{\partial}{\partial t}\left(u_{1}-u_{3}+h \frac{\partial w}{\partial x}\right)
$$




\subsubsection{Solution of the vibration equations}

To solve the equations of vibration, the displacement $u_{i}(i=1,3)$ and $w$ are solved to variable separation solutions with respect to time and location as shown by the following equations:

$$
u_{i}(x, t)=\bar{u}_{i}(x) e^{j \omega t}, w(x, t)=\bar{w}(x) e^{j \omega t}
$$

The equations (10a) to (10c) into which equation (12) is substituted are arranged with the following dimensionless quantities :

$$
\xi=x / L, W(\xi)=\bar{w}(x) / h, U_{i}(\xi)=\bar{u}_{i}(x) / L
$$

Moreover, the following newly defined variables are introduced.

$$
\begin{aligned}
& g=\frac{G_{2} L^{2}}{K H_{2}}, Y=\frac{K L^{2}}{D}, \Omega^{2}=\frac{\rho H \omega^{2} L^{4}}{D}, p=\frac{h^{2}}{L^{2}}, \\
& K=\frac{K_{1} K_{3}}{K_{1}+K_{3}}, k_{i}=\frac{K_{i}}{K}(i=1,3), c=\frac{C_{e q}(1-s)^{2} L^{2}}{K}
\end{aligned}
$$

The solutions of displacement $U_{i}(\xi)(i=1,3)$ and $W(\xi)$ are set as the following formation :

$$
U_{i}(\xi)=C_{i} e^{\lambda \xi}, W(\xi)=C e^{\lambda \xi}
$$

Vibration equations are modified by applying equations (11) to (15). Consequently, the following property equation is derived when it is assumed that the modified equation has any solution except.

$$
\lambda^{6}-\left(g_{a}+g_{b}\right) \lambda^{4}-\lambda^{2} \Omega^{2}+g_{b} \Omega^{2}=0
$$

where $g_{a}=p g Y s^{2}, g_{b}=g_{b 1}+\mathrm{j} g_{b 2}, g_{b 1}=g s^{2}$ and $g_{b 2}=c \omega$.

Six property solutions obtained by solving equation (16) are denoted as $\pm \lambda_{j}$ ( $j=1$ to 3 ), and the solutions of displacement $U_{i}(\xi)$ and $W(\xi)$ are given by the following equations with $\pm \lambda_{j}$ :

$$
\begin{aligned}
& U_{i}(\xi)=\sum_{j=1}^{3} a_{i j}\left[C_{j} e^{\lambda j \xi}-C^{{ }^{\prime}}{ }_{j} e^{-\lambda j \xi}\right] \\
& W(\xi)=\sum_{j=1}^{3}\left[C_{j} e^{\lambda j \xi}+C^{\prime}{ }_{j} e^{-\lambda j \xi}\right]
\end{aligned}
$$

where,

$$
a_{1 j}=\frac{1}{k_{1}} \frac{p g_{b} \lambda_{j}}{\lambda_{j}^{2}-g_{b}}, \quad a_{3 j}=-\frac{1}{k_{3}} \frac{p g_{b} \lambda_{j}}{\lambda_{j}^{2}-g_{b}}
$$

where $C_{j}$ and $C_{j}$ are undefined terms, which are decided for the solutions to meet the boundary conditions, including the state where one-side is fixed and the other side free, for example.

\subsection{Derivation of the equation to calculate the loss factor}

The loss factor $\eta$ of MRDC, which is a representative index of vibration damping performance, is calculated by the following equation with the solutions mentioned above:

$$
\eta=\frac{L_{f s} / 2 \pi+\eta_{2} P_{2}}{P}
$$

where, $P$ is the strain energy of the three layers given by equation (5); $P_{2}$ is the strain energy of the magnetic rubber layer given by equation $(6 \mathrm{~b}) ; L_{f S}$ is the frictional loss during one cycle of vibration; $\eta_{2}$ is the loss factor of the magnetic rubber layer obtained from experiment. Although the solutions obtained are imaginary, energy $P$ and $P_{2}$ are real values because they are calculated with the square of the length of the imaginary vector of the solution, which corresponds to a multiplication of the imaginary vector itself and its conjugated value.

$L_{f S}$ is given by the following equation, when it is considered that the frictional loss generated in the infinitesimal distance $d x$ during infinitesimal time $\mathrm{dt}$ is given by equation (8) :

$$
L_{f s}=4 \mu F_{M}(1-s) \int_{0}^{L}\left(u_{10}-u_{30}+h \frac{\partial w_{0}}{\partial x}\right) d x
$$

where the subscript 0 shows the real part of each displacement.

\section{Loss factor experiments}

In order to confirm the accuracy of the theory as set out in the previous chapter and to clarify the damping properties of MRDC, experiments were conducted by the resonance method.

\subsection{Specimens}

The specimens were beam-type MRDCs, 248mm long and $10 \mathrm{~mm}$ wide. In order to make clear the influence of material factors, their ferrite content was set at 40, 45, 50 and $60 \mathrm{vol} \%$, with the magnetic attractive force per unit area changed from $2.5 \mathrm{kPa}$ to the full magnetization value. The thickness of the constraining layer was from 0.3 to $10 \mathrm{~mm}$, while that of the magnetic rubber layer was from 1 to $6 \mathrm{~mm}$. Four base layers, each $250 \mathrm{~mm}$ long and $10 \mathrm{~mm}$ wide, were cut out from an SS400 steel block, as illustrated in Fig. 2. Their thicknesses were 2, 4, 8 and $16 \mathrm{~mm}$.

The loss factor and the resonance frequency were measured when each of the specimens mentioned above was attracted to the base layer.

\subsection{Method of experiment}

Figure 2 illustrates the measuring instrument. The body of each steel block, from which the base layers were cut, was fixed to a large size steel block, the other side

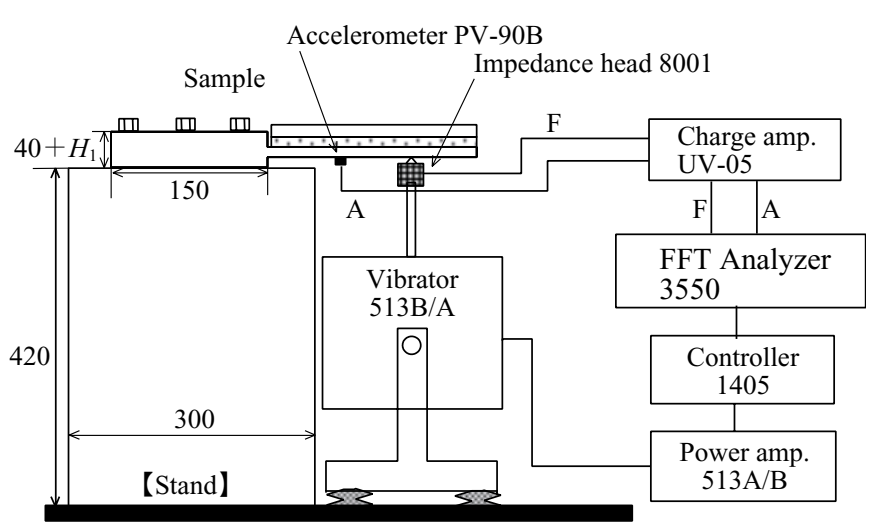

Size unit : $\mathrm{mm}$

Fig. 2 Measuring instrument for loss factor experiment 
of the base layer being free. For most specimens, the free side of the base layer was vibrated by a coil-type electric exciter in the form of a sweeping sine curve from $100 \mathrm{~Hz}$ to $3.2 \mathrm{kHz}$. The exciting force $F$ was measured by an impedance head attached on the upper part of the exciter, and the vibratory acceleration $A$ was measured by an accelerometer attached at a place $50 \mathrm{~mm}$ from the fixed edge of the base layer. The resonance curve of vibratory mobility $A / F$ was analyzed with an FFT analyzer, and loss factors at some vibration modes were calculated by dividing half the bandwidth frequency, that is the difference between two frequencies where the value of vibratory mobility is $3 \mathrm{~dB}$ less than that of the resonance frequency, by the resonance frequency.

\subsection{Calculation and measurement results}

The calculated and measured results at the 2 nd mode are discussed in this study because here the loss factor was found to be the highest in the measurable frequency range in both the theoretical calculation and actual measurement results for most specimens.

\subsubsection{Dependency on magnetic attractive force and fer-} rite content

Figure 3 shows the loss factor changes of MRDC at the 2 nd mode as the ferrite content was adjusted to 40 , 45,50 and $60 \mathrm{vol} \%$ for the different values of magnetic attractive force $F_{M}$. In Fig. 3, the thickness of the constraining layer and the base layer in all specimens was $2 \mathrm{~mm}$ and that of the magnetic rubber layer $3 \mathrm{~mm}$. From Fig. 3, it is revealed that the calculated MRDC loss factor results agreed with the measured data in most specimens, thus verifying the accuracy of the proposed theory. sult :

The following properties are recognized from this re-

(1) MRDC loss factor shows a peak against the magnetic attractive force. (2) MRDC loss factor increases as the ferrite content decreases when the magnetic attractive force is constant.

The reason why MRDC loss factor shows a peak against the magnetic attractive force is examined first.

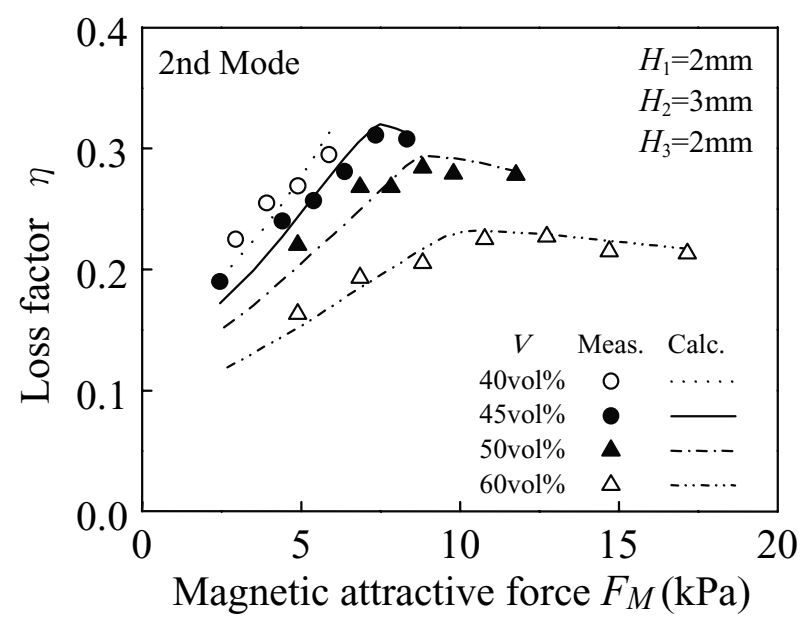

Fig. 3 MRDC Loss factor versus magnetic attractive force and ferrite content
The property is thought to originate from that of frictional loss because it plays an important role in MRDC. Frictional loss is defined as the product of frictional resistance and sliding displacement, as mentioned above. The frictional resistance increases and the sliding displacement decreases as the magnetic attractive force increases. A peak value arises as the effect of these contradictory factors.

Moreover, in the case of the specimen whose ferrite content was $40 \mathrm{vol} \%$, the magnetic attractive force is so small that it does not reach the ultimate peak loss factor value even when the specimen is fully magnetized.

Subsequently, the reason why MRDC loss factor increases as the ferrite content decreases is discussed. It is assumed that both the frictional loss and the internal loss would increase as the ferrite content decreased. The increases of frictional loss are considered to be due to the increases of frictional coefficient between the magnetic rubber and steel base layers, as the experimental results prove that it increases with decreases of ferrite content. Moreover, the inner loss is also thought to increase with decreases of the ferrite content because it is mainly caused by rubber deformation, which increases with higher rubber content and thus lower ferrite content.

\subsubsection{Dependency on thickness of three layers}

Figure 4 shows changes of MRDC loss factor at the 2nd mode when the thickness of the magnetic rubber layer was adjusted to 1 to $6 \mathrm{~mm}$ and that of the constraining layer to 0 to $5 \mathrm{~mm}$. In Fig. 4, the ferrite content of all specimens, each of which was fully magnetized, was $45 \mathrm{vol} \%$. From these results, it can be said that the calculated loss factor results deviate from the measured results when the thickness of the constraining layer is $5 \mathrm{~mm}$ and the thickness of the magnetic rubber layer is in the 1 to $6 \mathrm{~mm}$ range. From the results obtained for all specimens shown in paragraph 3.1 , it is also recognized that the error rate between the calculated and measured data remains small when the thicknesses of both the base layer and the constraining layer are less than $4 \mathrm{~mm}$. To estimate practically the applicable range of the theory, the range should be discussed from the viewpoint of the ratio between the thickness and length of each layer, because

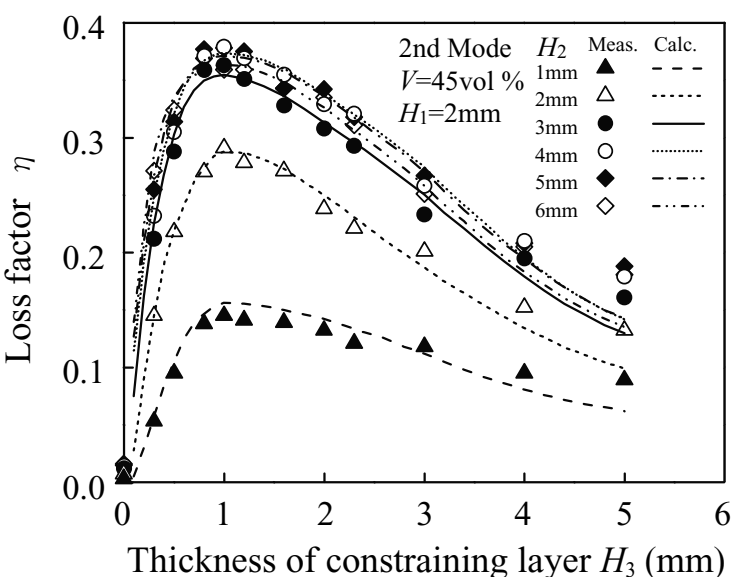

Fig. 4 MRDC loss factor versus thickness of constraining layer 
the accuracy of the flexural beam theory is generally related with the relationship between the thickness and beam length. From this, the applicable range of the proposed theory is estimated as being below 0.016 for the thickness of the constraining layer and base layers, and 0.004 to 0.024 for that of the magnetic rubber layer, times the layer length.

From Fig. 4, it can be also said that MRDC loss factor hits a peak value when the thickness of the constraining layer is approximately half that of the base layer, irrespective of the thickness of the magnetic rubber layer. As the results obtained for all specimens in 3.1 reveal, this property, or the fact that the ultimate value of the thickness of the constraining layer is sought at a point about half that of the base layer, is recognized irrespective of the thickness of the base layer $(2,4,8$ or $16 \mathrm{~mm})$.

Figure 5 shows MRDC loss factor changes at the 2nd mode when the thickness of the magnetic rubber layer was changed in the range of 1 to $6 \mathrm{~mm}$, that of the base layer was adjusted to be 2 or $4 \mathrm{~mm}$, and that of the constraining layer was $2 \mathrm{~mm}$. As a result, in both cases where the thickness of the base layer is 2 and $4 \mathrm{~mm}$, the MRDC loss factor increases simply with an increase of the thickness of the magnetic rubber layer in the range lower than $3 \mathrm{~mm}$ (0.012 times the layer length), and this tendency converges in the range over $3 \mathrm{~mm}$.

As a result of calculation and experiment, almost the same properties seen in the loss factor at the 2 nd mode mentioned above are witnessed during investigations into the $3 \mathrm{rd}$, 4th and 5 th vibration modes.

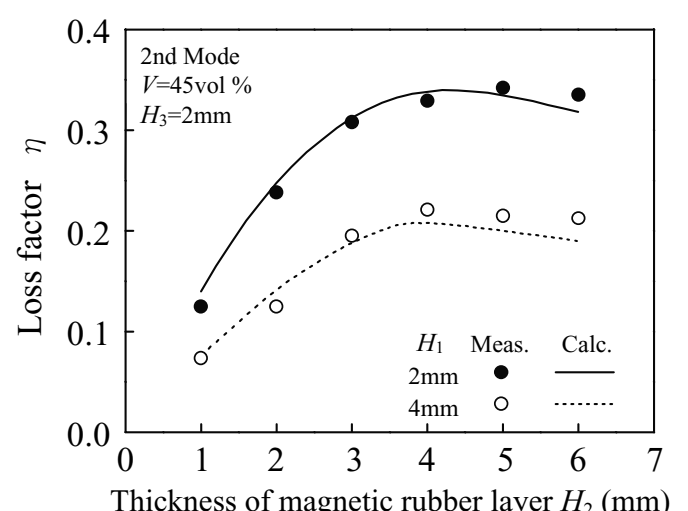

Fig. 5 MRDC loss factor versus thickness of magnetic rubber layer

\section{MRDC design optimization}

As mentioned above, it has been clarified that the proposed theory is accurate enough. As a result, it is now possible to apply this theory to the development of new products, and ensure optimal design at high efficiency.

As an example of optimal design of MRDCs, the following are the specifications obtained for those composed of the materials discussed in this paper or having a butyl rubber magnetic rubber layer and a steel constraining layer.

(1) MRDCs exhibit the highest damping performance when the ferrite content is $45 \mathrm{vol} \%$.

(2) MRDCs have an optimal value of magnetic attractive force (when the ferrite content is over $45 \mathrm{vol} \%$ ). It is not advantageous in actuality, however, to always manufacture MRDCs so that they have the optimal magnetic attractive force, since it requires precise magnetization during the manufacturing process, and often increases the manufacturing cost. On the other hand, the damping performance of MRDC is sufficiently high when the ferrite content is $45 \mathrm{vol} \%$ and the magnetic attractive force is $90 \%$ or over of the value of the fully magnetized. Accordingly, it is recommended that magnetizing rate is higher than $90 \%$ in consideration of these conditions.

(3) When the thickness of the constraining layer is half that of the base layer, MRDCs exhibit the highest damping performance. In practice, however, the thickness of the constraining layer is determined in consideration of various factors, including the required damping, weight limit not to obstruct installation, space at the installation site and material costs.

(4) The damping performance of MRDCs is adequately high, when the thickness of the magnetic rubber layer is $3 \mathrm{~mm}$ or over ( 0.012 times the layer length). In practice, however, it is recommended to make the thickness of the magnetic rubber layer 3 to $5 \mathrm{~mm}(0.012 \sim 0.015$ times the layer length) since the MRDC weight is desirably light enough to make the installation work easier, while ensuring appropriate damping performance.

\section{Conclusions}

In order to appreciate sufficiently the damping properties of the magnetic rubber damper with a constraining layer (MRDC), which consists of magnetic rubber and steel constraining layers, the flexural vibration of a beam covered with MRDC has been analyzed and experiments for the loss factor carried out. As a result, the following conclusions are obtained :

(1) The vibration equations of MRDC, which has frictional loss, are derived based on the Hamilton's principle expanded into a non-preservative system.

(2) A solution to the vibration equations of MRDC is derived by substituting the frictional resistance into a viscous resistance whose energy is the same as the frictional loss.

(3) Based on the analysis shown in (1) and (2), an equation to calculate MRDC loss factor, which is a representative index of the damping factor, is derived.

(4) The calculated loss factor results based on the proposed theory agree with experimental data in the range where the thicknesses of both the constraining layer and base layer are less than 0.016 and that of the magnetic rubber layer is 0.04 to 0.024 times the layer length.

(5) The relationships between MRDC damping performance and material factors, such as ferrite content, magnetic attractive force, the thicknesses of the magnetic rubber and constraining layers, are made clear by calculation and measurement results. Hence, after now, it is enabled to design MRDC efficiently with optimized damping properties by applying the proposed theory when new-type products are examined. 


\section{References}

1) SATO, H., HANSAKA, M., and MIFUNE, N.: "The development of Damping Properties of Magnetic-Vibration-Damper (MVD)," QR of RTRI, 38-2, pp.56 $60,1997$.

2) HANSAKA, M., et al.: "Prediction of Damping Property and Design for Optimization of Magnetic Rubber Damper with a Constraining Layer," Rep. of RTRI, 17-10, pp.41 47, 2002.

3) HANSAKA, M., and HAYASHI, I., et al.: "The Damping Property of Magnetic Rubber Dampers with a
Constraining Layer (1st, Derivation of the equation of loss factor based on the analysis of flexural vibration of beam)," Transactions-C of the Japan Society of Mechanical Engineers, 67-662, pp.25 31, 2001.

4) HANSAKA, M., and HAYASHI, I., et al.: "The Damping Property of Magnetic Rubber Dampers with a Constraining Layer (2nd, Estimation of the loss factor of magnetic rubber dampers with a constraining layer and experimental demonstration)," Transactions-C of the Japan Society of Mechanical Engineers, 67-666, pp.26 34, 2001. 